\title{
Validation of Neutron Diffraction and the Incremental Deep Hole Drilling Residual Stress Measurements of a High Strength T Butt Weld Test Piece Using the Contour Residual Stress Measurement Technique
}

\author{
Glen Sloan ${ }^{1, a^{*}}$, Xavier Ficquet ${ }^{2, \mathrm{~b}}$, Douglas Cave $^{2, \mathrm{c}}$, Karim Serasli ${ }^{2, \mathrm{~d}}$, \\ Ed Kingston ${ }^{2,}$, , Valerie Linton ${ }^{3, f}$
}

${ }^{1}$ School of Mechanical Engineering, University of Adelaide, North Terrace, Adelaide, Australia

${ }^{2}$ Veqter Ltd, Unicorn Business Park, Whitby Road, Bristol, BS4-4EX, UK

${ }^{3}$ Energy Pipelines CRC, Wollongong, Australia

aglen.sloan@bigpond.com.au, bxavier.ficquet@veqter.co.uk, cdouglas.cave@veqter.co.uk, dkarim.serasli.veqter.co.uk, ed.kingston@veqter.co.uk, ${ }^{\mathrm{f}}$ valerie.linton@epcrc.com.au

Keywords: Welding, Residual Stress, Neutron Diffraction, Contour Method, Stress Corrosion Cracking, Corrosion Fatigue, T Butt

\begin{abstract}
Off shore ring stiffened cylindrical structures are subjected to high stresses caused by static or variable external hydrostatic pressure and residual stresses due to the fabrication and welding processes. The use of numerical simulation allows a straightforward calculation of the stresses induced by the hydrostatic pressure in the structure. However it is more intricate to determine the residual stresses resulting from the progressive manufacture of components and assembly using a multi-pass welding processes. This paper presents the work carried out to ascertain the residual stresses present within a high strength welded T-plate test piece, representative of part of a ring stiffened cylindrical structure, by the contour method to determine the longitudinal residual stresses across the width of the test piece. This test piece had previously been subjected to neutron strain scanning in a pattern across the test piece and also the incremental Deep Hole Drilling (iDHD) and, the Deep Hole Drilling (DHD) processes to validate the neutron results for the longitudinal and transverse components of residual stress in the weld toe and centreline of the $\mathrm{T}$ butt weld. Therefore the work on this test piece is unique as three methods of residual stress measurement have been used. The results of these measurements are presented and compared to highlight agreements and discrepancies in the measured residual stresses by each method.
\end{abstract}

\section{Introduction}

Ring stiffened cylindrical structures operate in some of the worst environmental conditions particularly when they are used in naval platforms. These naval platforms will be subject to high stress low cycle fatigue due to change in depth. They are manufactured from high strength quenched and tempered steels and therefore are susceptible to welding induced defects such as Hydrogen Assisted Cold Cracking (HACC) for which the presence of tensile residual stresses is considered one of the driving forces for the crack development. These platforms also operate in environments where the microstructure of the quenched and tempered steels coupled with tensile residual stresses can result in Stress Corrosion Cracking (SCC) which can then initiate failure by corrosion fatigue. Therefore a comprehensive knowledge of residual stress profiles is required not just at the weld but any location where there is susceptibility to any of these failure modes. This knowledge of residual stresses becomes even more critical when proposing alternate welding details such as pass placement as per [1]. Fracture Mechanics assessment standards such as BS7910 do not address alternate pass placement strategies as discussed in [1], [2]. A research program to investigate these factors was 
implemented by the University of Adelaide with details of the work program presented elsewhere [2], [3].The test piece discussed in this paper had previously had strain measurements made using neutron diffraction (ND) on the Kowari strain scanner at the Bragg Institute, ANSTO, Sydney, Australia. The scanning pattern for this ND work is presented in [1] which is a sister paper for this conference. While comprehensive results were achieved across the base plate limited strain scanning results for the longitudinal direction were obtained in the fusion zone of the weld to the base plate and in the weld itself due to excessive beam path lengths as discussed in [1] and [2]. To partially overcome this two sets of iDHD/DHD measurements were undertaken as described in [1] and [2]. While these measurements gave additional information there were still critical gaps in knowledge, principally on the weld caps for sides 1 and 2 where it was not practical to undertake iDHD/DHD measurements on the entire weld cap. While more extensive DHD measurements could be undertaken the test piece would end up with numerous holes which could preclude use of other nondestructive or destructive techniques. Therefore it was decided to acquire additional residual stress information via use of the contour method.

\section{Experimental Methodology}

As detailed in [1] the longitudinal direction for plate rolling, curvature shaping and welding all coincided in the test piece which is standard practice for ring stiffened cylindrical structures. In addition, the longitudinal stress profiles across all areas of the base plate and web are the ones that show the most dramatic change but for which it is hardest to gather information by the ND method particularly in the region under the $\mathrm{T}$ butt weld. Therefore the decision was made to undertake sample contouring by cutting transversely across the test plate and therefore causing relaxation in the longitudinal direction. The contouring residual stress work was undertaken by Veqter Ltd using the methods described on their web site [4]. The cut was undertaken using the electro discharge method at a location greater than $30 \mathrm{~mm}$ from the closest pre-existing DHD site. The relaxed profile was measured using CMM on a grid profile of $0.5 \mathrm{~mm}$. As detailed in [1] the relatively short weld length enabled each weld pass to be undertaken without stops or starts along the length of the weld which would have involved other activities such as grinding. Therefore there is consistency in residual stress profiles along the length. In addition the welders practiced extensively achieving constant heat input levels [2]. The heat input levels for the passes was $1.7 \pm 0.1 \mathrm{~kJ} / \mathrm{mm}$. Therefore correlation between residual stress levels along the length of the test piece determined by the different methods was assured.

\section{Results}

A pictorial representation of the results are presented in Fig 1. The areas of the base plate away from the region of the web and the $\mathrm{T}$ butt weld show tensile regions towards the concave surface of the base plate with a zigzag pattern of tensile and compressive zones through the base plate. These extend up to the HAZ and fusion zones of the T butt weld noting that the test piece had reduced weld caps flush with the faces of the abutting web stiffener. The contour plot clearly shows that the residual stress of the first pass of the capping run side 2 i.e. at the intersection of the base plate and the web, see Fig 2 is compressive. This is not the case for side 1 as the equivalent weld pass has low tensile residual stresses. Compressive residual stresses are present on both sides of the weld for the capping passes up to the last capping pass on both sides where they are tensile. For these passes the residual stresses are up to 0.25 of the nominal minimum yield strength of the weld metal. The highest tensile stresses have occurred towards the center of the fusion zone in the base plate and at the root region of the full penetration $\mathrm{T}$ butt weld. The residual stresses in the web away from the weld region quickly drop to low compressive values. The residual stress profiles to be addressed in the discussion section are indicated by the red lines shown on the cross sectional plot in Fig.1. 


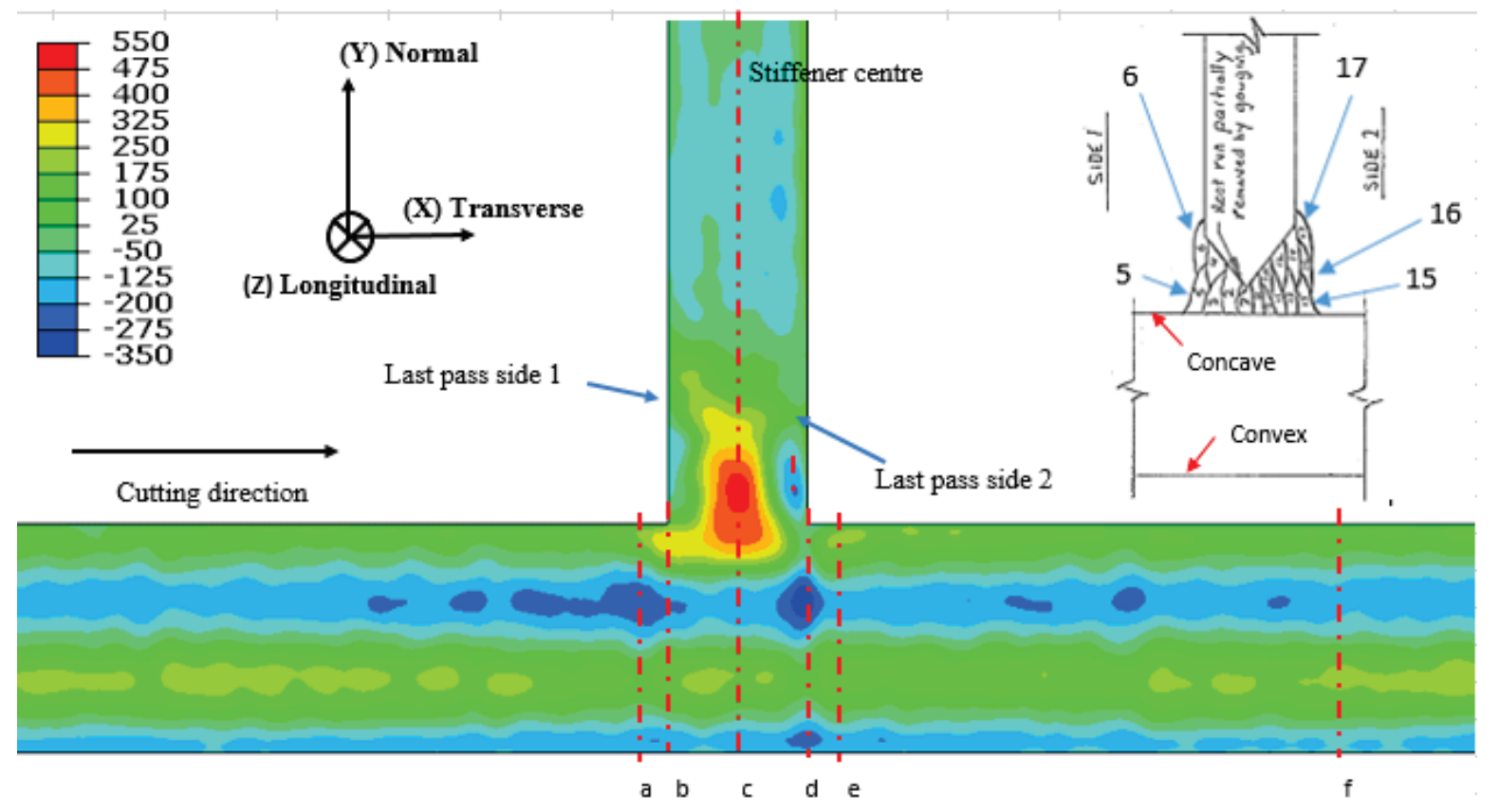

Fig. 1 Output from the FEA analysis of undoing the measured surface profile. The residual stress profile generated is for the longitudinal direction relative to the welding. The insert shows pass placements and the dashed red lines, a to f, are through thickness profiles which will be discussed.

\section{Discussion}

Throughout the following discussion section the datum for the position of each measurement by all methods will be changed to that which was used in [2] for the neutron diffraction testing. The initial areas of discussion will effectively be a sanity check for the comparison of the contour results against the neutron diffraction results for areas away from the $\mathrm{T}$ butt weld itself. These will then be followed by the more complicated areas in way the weld.

The first location of interest is the through thickness residual stress profile at $90 \mathrm{~mm}$ from the centerline of the web on side 2 of the weld, line f Fig.1. In Fig 2 comparative plots of the longitudinal residual stress profiles determined by the neutron diffraction technique with those from the contour method. In addition a plot of the neutron diffraction results from Pearce [3] on a curved plate segment shaped to the identical radius, and in fact from the same shaped plate used for the base plate of this test piece has been superimposed on these results together with the theoretical longitudinal residual stress profile for the shaped base plate calculated by the formulas given in Kendrick [5]. As can be seen in Fig 2 there is correlation between the results as they all show the zigzag pattern through the base plate for a shaped plate to a radius with tensile residual stresses towards the concave surface and compressive residual stresses towards the convex surface. Also the magnitude of the near surface residual stresses is less than that of the through thickness residual stress peaks and therefore corresponds with the results of Pearce [3] and calculations from Kendrick [5]. The kink upwards in the longitudinal residual stresses near the convex surface determined by the contour method cannot be explained at this stage as it is well below any surface induced profile from activities such as shot peening. The errors for the residual stresses determined by ND for all plots are given in [1] and for the DHD/iDHD are $25 \mathrm{MPa}$ maximum. 
Through Thickness Longitudinal Residual Stresses $+90 \mathrm{~mm}$ Side 2 of Weld

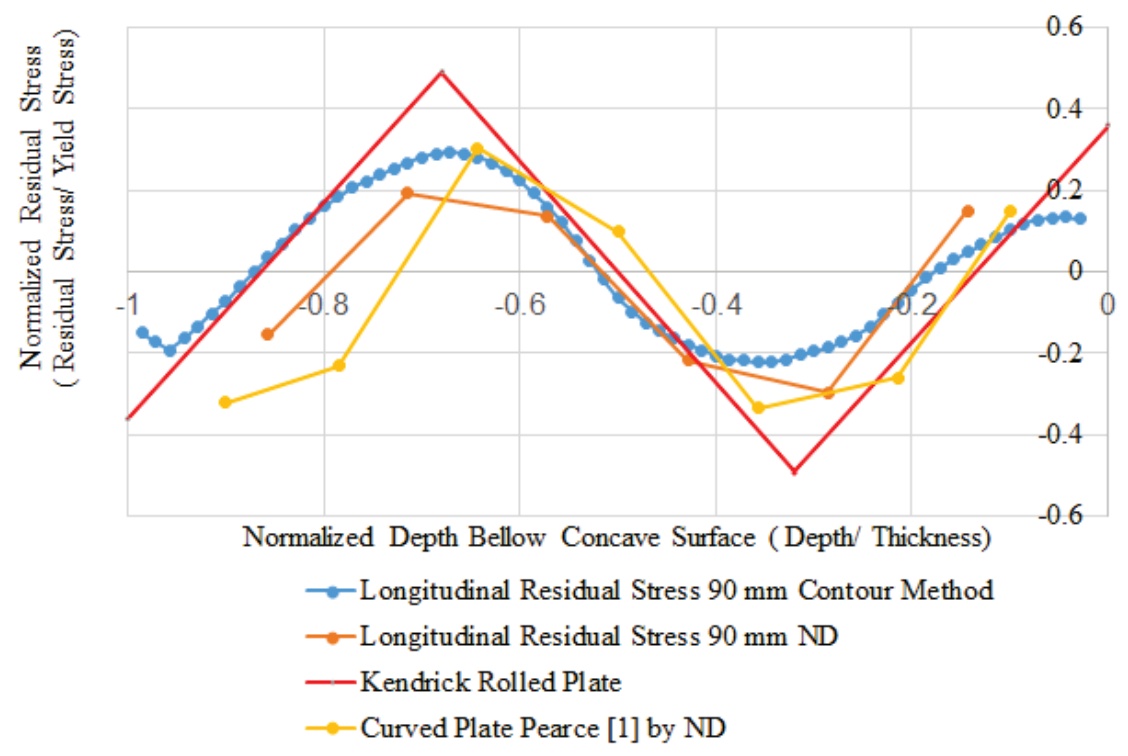

Fig. 2 Plots of longitudinal residual stress through the base plate by various methods at $90 \mathrm{~mm}$ from centerline of the web member. All closely match the theoretical profile developed by Kendrick [5]

The next locations of interest are the through base plate profiles $5 \mathrm{~mm}$ either side of the face of the abutting web member, lines a and e Fig 1. These locations are clear of the HAZ and Fusion Zones of the weld. The residual stress profiles obtained by the contour method are shown in Fig. 3 with the results from the neutron diffraction testing down these two paths superimposed. While at first glance there appears to be little change from the profile shown in Fig. 2 there are subtle differences. The through thickness profiles show a zigzag pattern starting with tensile residual stresses near the concave surface and compressive residual stresses near the concave surface of the base plate similar to those determined at the +90 through base plate location side 2 of the weld the magnitudes of the peaks have changed. The plots from contouring now have the magnitude of the peak stress near the concave surface greater than the through thickness peak. In addition the levels of tensile stresses are reducing rapidly at the surface whereas the profile at $\pm 20 \mathrm{~mm}$ (not shown) do not reflect this and therefore establishes that the welding has affected only a small distance either side of the abutting member.

The profiles through the centerline of the web as determined by ND, iDHD/DHD and by Contour Method are given in Fig 4. A sanity check on the contour results with respect to possible significant variation across the equivalent gauge volume, i.e. $5 \mathrm{~mm}$, of the DHD measurements was undertaken and showed that there was insignificant variation in the base plate and minor variation in the weld region. The variation in the weld region was equivalent to the apparent variation in residual stresses by the two methods at the weld root region. While this indicates that there is variation between the two destructive methods in the base plate there is commonality in the weld region. The main thing that is shown is that the two methods have established that there is a high tensile residual stress levels in the region of the centerline of the web/weld. While the neutron diffraction profile in the base plate does not directly correspond with those from the other two methods it follows the general trend. While no longitudinal residual stresses in the weld region could be obtained by this method along this line the transverse and the normal strains were determined and were tensile [2]. The ND full cap individual result [1], [2] corresponds closely with the DHD and Contouring results increasing confidence in the profiles obtained. 


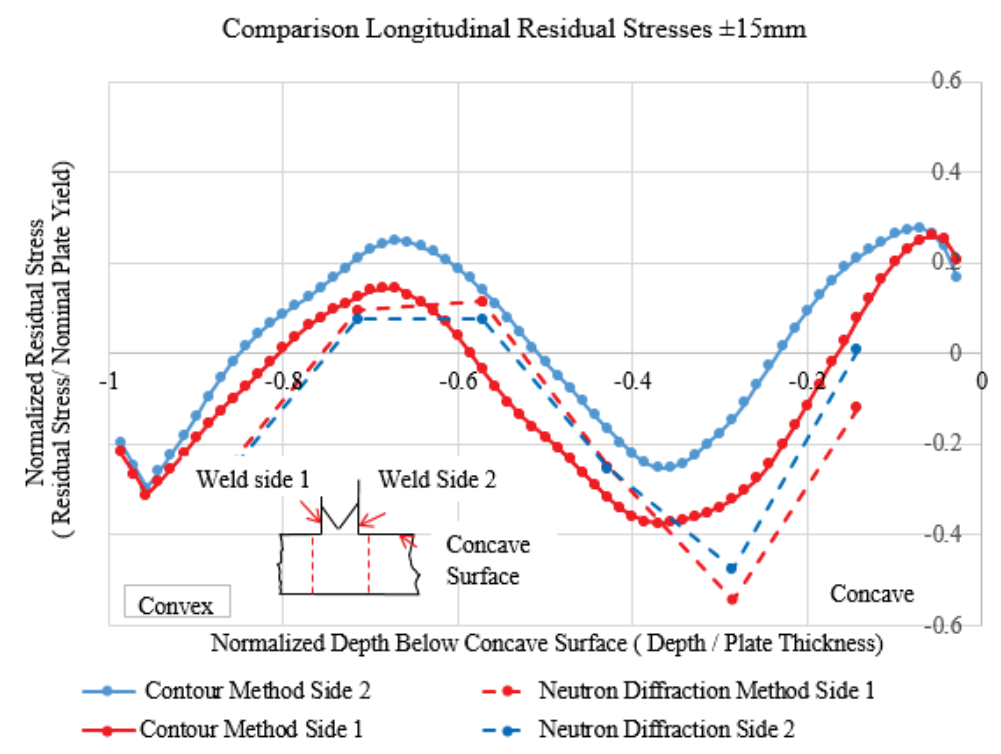

Fig. 3 Comparison plot of longitudinal residual stress profiles through the base plate at $\pm 15 \mathrm{~mm}$ from the centerline of the web. Profiles show the slight alteration from Fig 2 due to the effect of the weld.

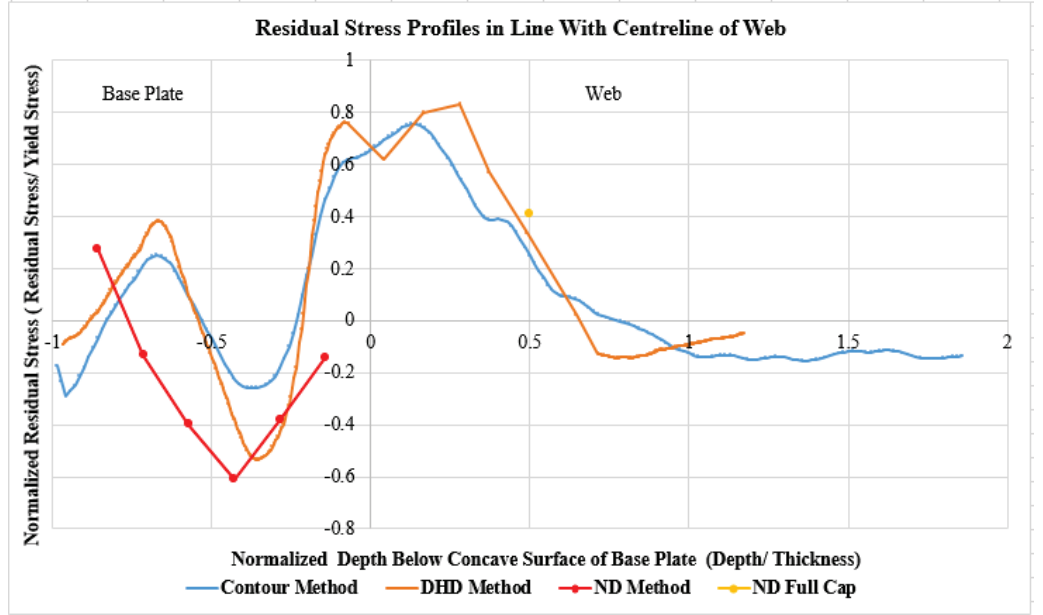

Fig. 4 Comparative plots of longitudinal residual stresses through the base plate and web centerline determined by 3 methods.

Turning to the results presented in Fig 5 for the weld toe region side 2 the results from all methods show that there is a major drop in the longitudinal residual stress magnitude at the weld toe and in the subsequent passes of the weld cap. Of particular interest is that the single ND result in the weld cap corresponds closely with the equivalent profile obtained by the contouring method both for the $8 \mathrm{~mm}$ and $10 \mathrm{~mm}$ line. All profiles show a drop in the residual stresses at the weld toe region down to low tensile or compressive. This confirms that the alternate pass placement strategy has achieved its aims namely reducing the risk of SCC and HACC at the weld toe regions where defects are subjected to high stress low cycle fatigue. As noted in [1] the results for the DHD test centered $11 \mathrm{~mm}$ from the centered of the web and therefore $1 \mathrm{~mm}$ of the face of the web are more closely aligned to the ND results for $+15 \mathrm{~mm}$ from the center of the web, $5 \mathrm{~mm}$ away from the face. They still show a drop in magnitude at the weld toe. The residual stresses at the weld toe on side 1, line b Fig1 (not shown) have been reduced to low magnitude tensile stresses and therefore reducing risk of HACC and SCC. 


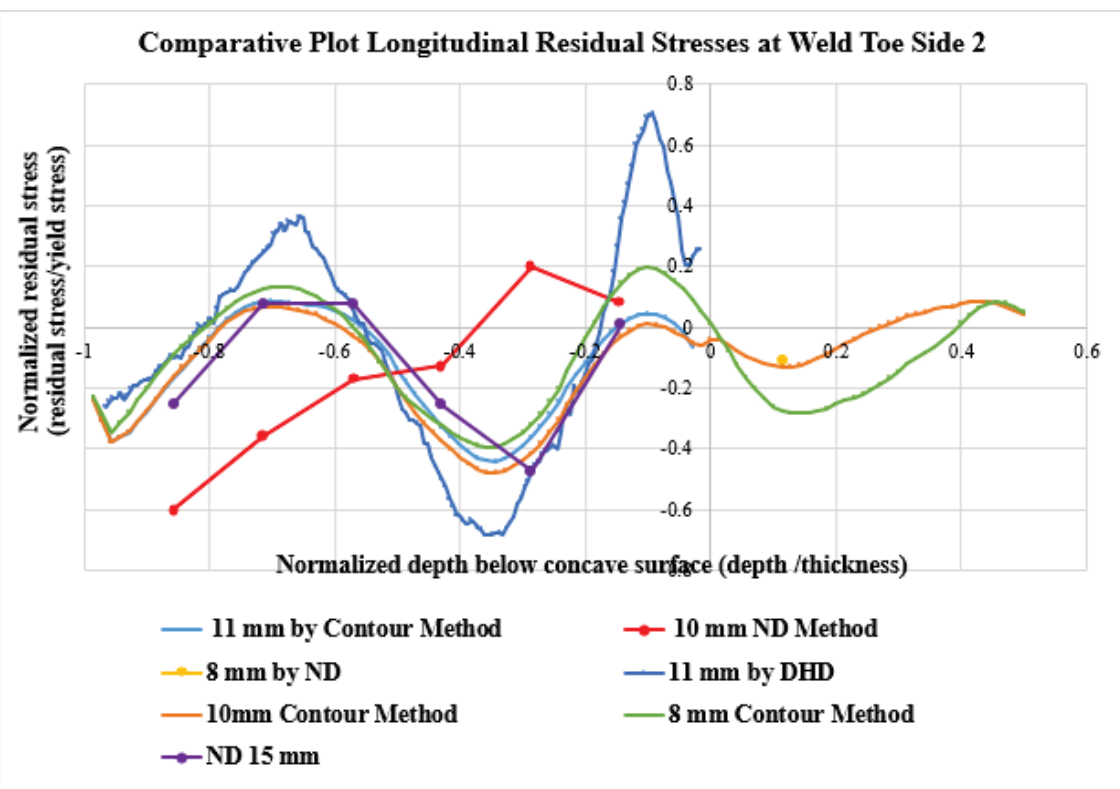

Fig. 5 Comparative Plots of longitudinal residual stresses through weld toe region side 2 of weld determined by 3 methods.

\section{Summary}

The additional longitudinal residual stress information obtained by the cutting method has enhanced the understanding of what is the impact of the specific pass placement strategy shown in Fig 1 for a $\mathrm{T}$ butt weld. The results obtained by the contour method confirm those previously obtained by the ND and DHD methods in that the passes in the capping runs towards the base plate and abutting weld member toes are altered to have compressive residual stresses. The same applies to the base plate at the weld toe side 2 . Therefore the pass placement strategy progressing away from the base plate reduces the risk of SCC at the weld toe region and in the weld caps. Similarly the risk of formation of HACC defects in the weld cap and weld toe region will also be reduced as the residual stresses are reduced. The high tensile residual stress region has been moved to the center of the weld. Therefore any defects forming in this region will be embedded away from the weld caps and are more easily detected by non destructive testing.

\section{Reference}

[1] G. W. Sloan, V. M. Linton, O. Kirstein, X. Ficquet, E. Kingston, I Brown Residual Stress Measurement in a High Strength T Butt Weld Specimen by the Neutron Diffraction and Deep Hole Drilling Techniques, ICRS 10 Sydney 2016.

[2] G. W. Sloan, "The influence of welding variables on the residual stress profile around T butt and MMAW butt welds in thick section high strength steel”, Master's Thesis, University of Adelaide 2016.

[3] S. Pearce, "Breakdown of residual stresses in highly restrained thick section welds", Doctoral thesis, University of Adelaide, 2016.

[4] Information on http://www.veqter.co.uk

[5] S. Kendrick, "Structural design of submarine pressure vessels", NCRE report R483, March 1964. 